\title{
Computerized practice tests and effects on in-class exams
}

\author{
W. H. LEE-SAMMONS and KEITH A. WOLLEN \\ Washington State University, Pullman, Washington
}

\begin{abstract}
Twenty-eight introductory psychology college students volunteered to learn a technique that might help improve their in-class, multiple-choice exam performance. Two 12-question multiplechoice tests with detailed feedback were presented on a personal computer. There were two types of feedback. Separate feedback consisted of a brief statement indicating why a specific answer was either right or wrong. Combined feedback consisted of the four separate feedbacks combined into a single paragraph. Eighteen students from another introductory class, who did not get to participate in these practice tests, served as a control. A within-group comparison of the two feedback formats showed differences in degree of improvement dependent on the level of achievement by students on the first in-class exam. When compared with data from the control group, the results show significant improvement by the low-level participants on the second in-class exam $(p<.01)$.
\end{abstract}

The purpose of the present study was to determine if interactive detailed feedback, presented by means of computerized practice tests, could improve in-class test scores of low-achieving students. Techniques for examining the potential success of feedback are limited. Moreover, many instructors have few means of determining how to improve students' test performance, and in addition, they have limited time to do so personally. The present research investigates a practice-test technique that requires little or no time on the part of the instructor.

Carter (1984) outlined feedback as having four characteristics: function, timing, schedule, and type. The present research is concerned with type. Feedback type ranges from the simple presentaton of results to viewers, to the presentation of detailed and elaborate messages that provide or direct one to explanations. Type of feedback has been found to be important in high-level cognitive tasks such as the diagnosis of myocardial infarction (Anderson, Kulhavy, \& Andre, 1971).

Generally, feedback type can fall into any one of, or any combination of, the five following categories: (1) no feedback; (2) knowledge of result (correct or wrong); (3) knowledge of result and of the correct response; (4) knowledge of result and of the correct response, plus an explanation (KCRE); and (5) knowledge of result and of the correct response (indicating an incorrect response), plus an explanation (KIRE). Specifically, KCRE involves information concerning: (1) the choice selected, (2) the correct choice, and (3) an explanation of why the correct choice was correct. The KCRE and KIRE types differ only

Correspondence may be addressed to W. H. Lee-Sammons, Department of Psychology, Washington State University, Pullman, Washington 99163 in terms of which choice is explained (the correct or the incorrect one). The combination of KCRE and KIRE feedback has been coined interactive teaching (Roper, 1977).

Recent studies of programmed instruction as an interactive teaching device show that the presentation of a mixture of feedback types is better than a mere evaluation of student performance (Carrier \& Sales, 1987; Merrill, 1987). Furthermore, a mixture of feedback types has been shown to be more effective than only one type, particuarly in reducing the number of incorrect selections when students select choices on introductory psychology muliple-choice tests (Metzger, 1985; Pettijohn, 1985) and on other science-based material (Carrier \& Sales, 1987; Merrill, 1987).

The practice tests in the present study involved the combination of KCRE and KIRE feedback in either of two formats. A separate feedback consisted of a brief statement indicating why a specific choice was correct or incorrect. A combined feedback consisted of the four separate feedbacks combined into a single paragraph. Providing feedback in a combined fashion requires more effort on the part of the students in order to tie specific portions of the feedback to specific choices. Feedback presented in smaller, separate, and more manageable units may work better for low-achieving students. Conversely, the more complex combined task may be better for high achievers. In the present study, the two feedback formats were used to determine which one would be more effective for low-achieving students.

Our goal was to determine if computerized practice tests could increase the performance of students on an in-class exam. It was predicted that students taking practice tests with KCRE and KIRE feedback would improve in-class test scores. Furthermore, it was also predicted that this would occur possibly because the explanations would 
help the students (1) note relationships between terms, (2) learn to identify key words in questions, (3) become more motivated to study, and (4) learn test-taking skills.

\section{METHOD}

\section{Subjects}

Sixty-two students enrolled in one of two sections of introductory psychology volunteered to participate in this experiment. Of the 62,44 students were from one section $(n=91)$ and 18 from another $(n=56)$. Of the 44 students, the 20 females and 8 males who actually participated constituted the experimental group. The control group consisted of the 18 students who signed up from the other class section. The 5 females and 11 males from the first section who signed up but failed to appear for the sessions constituted the experimental no-show group.

\section{Materials}

Questions. All test questions came from the test bank provided by the pubiisher of the text Psychology (2nd ed.; Crider, Goethals, Kavanaugh, \& Solomon, 1986). Ory (1986) developed the test-bank program on the basis of Bloom's Cognitive Taxonomy of Educational Objectives (1956). The questions in the bank were organized into three distinct types: (1) factual, (2) conceptual, and (3) applied.

The chapters in Crider et al. were subdivided into units on the basis of major headings. The number of questions per unit was approximately proportional to the number of pages in a unit. Insofar as possible, an attempt was made to equate the number of factual questions with the combined number of conceptual and applied questions.

In-Class Exam 1. The questions on Exam 1 covered chapters 1,2 , and 3 in the text. There were 43 questions on the experimental group's test and 53 questions on the control's. The tests had 36 multiple-choice questions in common. The 36 questions consisted of 16 factual, and 20 conceptual or applied types. The remaining questions were based on the lectures by each instructor. The students were allowed to omit three questions.

Practice tests and In-Class Exam 2. The questions covering chapters 5 and 6 were selected in pairs. Both members of a pair were of the same type, factual or conceptual and applied; both dealt with material appearing under the same inajor heading in the text. The questions were randomly assigned so that one question of each pair appeared on the practice test and the other on In-Class Exam 2.

One practice $t$ st, which covered material from chapter 5, consisted of eight factual and four conceptual or applied questions. The second practice test, which covered material from chapter 6 , consisted of six factual and six conceptual or applied questions.

The questions on in-class Exam 2 covered chapters 5, 6 , and 7 in the text. There were 53 questions on each class section's exam. As with Exam 1, both classes had 36 questions in common, and the students were allowed to omit 3 questions. The 36 questions consisted of 12 questions from each of the three chapters. In addition to the 36 questions on the chapters, each exam contained 17 lecture questions.

Feedback. On each test, feedback was structured to provide the students with elaborative information about right and wrong answers. For example, in a question about operant conditioning in which "generalization" had been selected as the answer, the feedback explained (1) what "generalization" was, and (2) why it was or was not an appropriate selection for that particular question.

Design. The design consisted of feedback format (separate or combined) and student-achievement level (low or high) as the between-groups factors. Achievement level was determined by performance on Exam 1 test-bank questions. The students with positive $z$ scores made up the high-level group, and the students with negative $z$ scores made up the low-level group. Performance on questions on text chapters was the dependent variable. This measure had four within-group levels. The first level included scores on Exam 1 questions from chapters 1, 2, and 3. Levels 2, 3, and 4 consisted of scores on Exam 2 from chapters 5, 6, and 7, respectively.

Questionnaire. A 14-item questionnaire was given to determine confidence, study time, and overall feelings about the practice-test procedure. The first 13 statements followed a four-level Likert scale. Thus, statements such as "I read chapter six before the second mini-test" appeared on the questionnaire, and the students were to respond on a scale ranging from "strongly disagree" through "disagree" and "agree" to "strongly agree." The 14th question asked students to predict how many practice-test questions they would answer correctly (see Appendix C).

\section{Procedure}

The students signed up, in class, to attend one of four sessions held in a microcomputer laboratory reserved for this occasion. The students took a 30 -min practice test in each of 2 consecutive weeks. Immediately before the beginning of the second practice test, the questionnaire was distributed. Each practice test was given during the week when the material that it covered was taught in class.

Upon entering the lab, the students were randomly assigned to one of 15 workstations. The students received the same feedback format in both practice tests. All the students started at the same time, had $30 \mathrm{~min}$ to complete the task, and were monitored. They were told to read the information presented on the screen and to work at a comfortable pace.

The two practice tests were presented on IBM XT microcomputers. To start each practice test, the students were told simultaneously to type "begin."

Practice-test presentation. Each practice test was presented twice. During the test phase, the questions appeared in the format of a stem followed by 4 choices. The 
students read each question and selected a choice by pressing the appropriate key on the keyboard. Following each selection, a new question appeared on the screen; no feedback was presented. This procedure continued until all 12 questions had been answered.

The second presentation, the feedback phase, started immediately after a choice to question 12 had been selected. At this point, the first question reappeared on the screen and the feedback phase was presented in one of two formats, separate or combined. The time students spent during the feedback phase was recorded for both formats.

Separate format. In the separate format, an incorrect choice was followed by a short explanation of why that particular selection was incorrect (KIRE). If an incorrect choice was selected, students read the feedback and were then asked to pick another choice. If the second selection was incorrect, feedback was presented that explained why it was incorrect. This continued until the correct choice was selected, whereupon KCRE was presented.

If the students had selected the correct choice during the test phase, the following occurred during the feedback phase: Immediately after an explanatory statement or KCRE, a statement appeared asking if they wanted to know why other choices were incorrect. If so, the students simply selected an incorrect choice, and read the feedback for that answer; if not, they pressed any key to continue to the next question. In this fashion, they could view feedback for $0,1,2$, or all 3 incorrect choices.

Upon completion of all 12 questions, the students received a printout of the results. The printout indicated (1) the number and percentage of questions answered correctly, (2) the percentages of factual and conceptual/applied questions answered correctly, (3) the total time spent during the test phase, (4) the time spent on each question during the test phase, and (5) pages in the text to review. The pages for review were the pages for the units with respect to which questions had been answered incorrectly.

Combined format. The students who had the combined format received the feedback immediately after the second presentation of the question and choices, whether right or wrong the first time through. For example, if a student had selected a choice correctly during the test phase, combined feedback was presented immediately after the question and choices appeared at the start of the feedback phase. After reading the feedback, the student continued on to the next question by hitting the space bar. This procedure continued until all 12 questions were presented. When finished, the student received the same type of printout as in the separate format.

\section{RESULTS AND DISCUSSION}

The performance on Exam 1 test-bank questions was used to determine the achievement levels of all the students. The number of questions answered correctly was changed to percent correct, and these raw scores were converted to $z$ scores to standardize the values. It was anticipated that the students in the lower part of the class would show the largest gains from the practice test. Accordingly, the students with negative $z$ scores $(n=10)$ were considered as one group (low level), and the students with positive $z$ scores $(n=18)$, were considered as another group (high level).

\section{Separate versus Combined Feedback}

A three-way analysis of variance was performed on the separate- as opposed to the combined-feedback data presented in the first four rows in Table 1. Format type and achievement level were the two between-groups factors. The within-group variable, tested material, had four levels. The performance on Exam 1 constituted Level 1. The performances on the three chapters comprising Exam 2 made up the other three levels. The questions on the lectures were ignored because they differed between the two classes.

The three-way interaction (format type $\times$ achievement level $\times$ tested material) was not significant $[F(3,72)=$ $1.81, p<.11]$. The test of the two-way interaction of achievement level $\times$ tested material was significant $[F(3,22)=6.73, p<.05]$, indicating a need to examine the simple effects.

Collapsed over chapters, the mean scores for low-level separate-feedback subjects were significantly increased from Exam 1 to Exam $2[-0.59$ vs. $0.28 ; t(5)=2.61$, $p<.05]$. Similarly, scores for the low-level combinedfeedback subjects also increased $[-0.81$ vs. $-0.13 ; t(3)$ $=2.75, p<.05]$. The predicted advantage for separate feedback over combined was obtained. The separate feedback for the high-level students had the opposite effect,

Table 1

Mean $z$ Scores of Computer-Trained Students, Control Volunteers, and Experimental No Shows

\begin{tabular}{|c|c|c|c|c|c|}
\hline \multirow[b]{3}{*}{ Groups } & \multirow[b]{3}{*}{$n$} & \multicolumn{4}{|c|}{ Test Bank Questions } \\
\hline & & \multirow{2}{*}{$\frac{\text { Exam 1 }}{\text { Chapters 1-3 }}$} & \multicolumn{3}{|c|}{ Exam 2} \\
\hline & & & Chapter 5 & Chapter 6 & Chapter 7 \\
\hline \multicolumn{6}{|c|}{ Separate } \\
\hline Low & 6 & -0.59 & 0.02 & 0.34 & 0.44 \\
\hline High & 8 & 0.76 & 0.31 & -0.21 & -0.01 \\
\hline \multicolumn{6}{|c|}{ Combined } \\
\hline Low & 4 & -0.81 & -0.08 & -0.27 & 0.05 \\
\hline High & 10 & 0.70 & 0.66 & 0.65 & 0.81 \\
\hline \multicolumn{6}{|c|}{ Control } \\
\hline Low & 10 & -0.83 & -0.76 & -0.98 & -0.91 \\
\hline High & 8 & 0.73 & 0.44 & 0.55 & 0.41 \\
\hline \multicolumn{6}{|c|}{ No Shows } \\
\hline Low & 11 & -0.78 & -1.02 & -0.94 & -0.99 \\
\hline High & 5 & 0.12 & 0.19 & 0.33 & 0.24 \\
\hline
\end{tabular}

Note-There were 6 females and 4 males in the low-level computertrained groups, 5 females and 5 males in the control group, and $4 \mathrm{fe}$ males and 7 males in the no-show group. 
decreasing significantly $[0.76$ vs. $0.04 ; t(7)=2.56$, $\beta<$. OS]. There was no change from Exam 1 to Exam 2 for the high-level subjects having combined feedback (0.70 vs 0.71 ).

\section{Low-Level Training versus Low-Level Control}

The data for the training and control groups are presented in Table 1. Rows 1 and 3 represent performance by the low-level participants of the treatment group (training). The data for low-level subjects from the other class section (control) appear in row 5.

The main effect of training revealed a significant difference tetween Exam 1 and Exaza 2 for the low-level craining group $[F(1,18\}=12.83, p<.05]$. The analysis of the main effect of tested material did nol meet significance $[F(3,54)=1.82, p>.05]$. Thus, the control group revealed no evidence for a change from Exam 1 to Exam 2.

\section{Learning, Test-Taking Skills}

A reasonable interpretation for the effect of training generalizing to chapter 7 is that the studerits may have learned test-taking skills and applied them to material not covered on the practice test. This interpretation is especially plausible if we compare the chapter 7 question performance of the low-level training group with that of the low-level no-show, or control group (see Table 1).

We had anticipated an increase in Exam 2 performance on the part of the low-level training group. The chapter 7 results, however, suggest that a considerable portion of the overall increase can be attributed to performance on the chapter 7 questions. This is especially noteworthy, since the practice tests did not include any questions from chapter 7 .

That is, if the practice tests were aiding students only on the chapeers covered on the practice tests, we would expect no, or very little, increase in performance on chapter 7 guestions. The low-leyel no-show and control group chapler 7 date support the notion of no increase in performance. Therefore, it is plausible that the low-level training sndents had perhaps learned ways to snudy chapter 7 and thus prepare themselves for that portion of the in-class exam.

\section{Less Likely Interpretations}

Feedback viewing time. One unlikely interpretation of the different effects among the high- and low-level subjects is that the low-level students spent more cime on the material. Since the students who received separace feedback could monitor how many answers they chose to Ieview, whereas the ones receiving combined feedback could not, one could argue that the separateness of feedback was confounded with the decision to receive it. This is a plausible assumption, and if it were true, it would be reasonable that they would have profited more from the practice tests. However, this did not occur. There was no significant difference in viewing time. Hence, it is unreasonable to argue that facilitation occurred as a function of reviewing time.
Regression toward the mean. Another possible but improbable isiespsetation for the increase in performance of the low-level subjects and decrease in performance of the high-level subjects on Exam 2 questions is regression toward the mean. Several lines of evidence argue against such an interpretation. If regression occurred, it should have appeared in the experimental no-show group. The data for the no-show group, found in the last two rows in Table 1, show no evidence of regression. Likewise, there was no evidence of regression for either low- or high-level subjects in the control group (see rows 5 and 6 in Table 1).

Finally, regression soward the mean would not accoun for the low-Jevel separate-feedback subjects who increased performance above the mean or for the high-level combined-feedback subjects who showed no regression at all. Consequently, it is reasonable to reject regression.

Similar question exposure. Another unlikely interpretation of the increased performance by the low-level achievers in the training group is that the practice tests aided the students by familiarizing them with specific content covered on the in-class exam. As has been mentioned, special attention was given to selection of the questions in order to reduce the possibility of familiarizacion.

Furthenore, the effect of taining on chapters 5 and 6 generalized 10 chapter 7 . Since the effect on chapter 7 was even larger than for chapters 5 and 6 , and since chapter 7 had no practice test, it cannot be argued that the observed increase resulted form exposure to material presented during the practice tests.

\section{Questionnaire}

The questionnaire was given to examine the students' opinions of the technique and to determine if they agreed it had influenced cheir study behavior and their confidence in doing well. Overall, the questionnaire data agree with the results of Petritotin (1985), who found that students rated a praclice-1est procedure very highly and who also reported that the students thought it had helped them leam how to prepare for tests.

\section{Predicted In-Class Exam Scores}

It was of interest to see if low-level practice-test participants increased their in-class test-taking confidence. Therefore, students were asked to predict their Exam 2 scores immediately before taking the in-class exam. No significant difference was obtained. Thus, the experimental subjects and controls made the same predictions, hut in actuality, the experimental subjects fid better.

\section{FINAL CONCLUSIONS}

In this study, we were primarily interested in the effect of the practice-test procedure on low-level volunteers. However, it was a bit surprising that the high-level group did not seem to benefit from this procedure. The combined feedback, collapsed over chapters, had no effect on high-level students $[t(9)=0.03, p>.05]$, and the 
separate feedback had a significant negative effect $[t(7)$ $=2.34, p<.05$ ].

Perhaps the high-level students considered the practice test more of an assessment technique than an instructional tool. If so, one explanation is that the low-level students reviewed the material suggested in the printout and the high-level students did not. Another possibility is that the high-level students' study skills were already better or more firmly entrenched and therefore not easily modified by the computer task.

In conclusion, it appears that the practice task can increase the performance of low-level students on an inclass multiple-choice exam. Such an increase may arise from their increasing motivation to study and their increasing abilities at test-taking.

\section{REFERENCES}

Anderson, R. C., Kulhavy, R. W., \& ANdre, T. (1971). Feedback procedures in programmed instruction. Journal of Educational Psychology, 62, 148-156.

BLoom, B. S. (Ed.). (1956). Taxonomy of educational objectives. New York: David McKay.

Carrier, C. A., SAles, G. C. (1987). Pair versus individual work on the acquisition of concepts in a computer-based instructional lesson. Journal of Computer-Based Instruction, 14, 11-17.

CARTER, J. (1984). Instructional learner feedback: A literature review with implications for software development. Computing Teacher, 10, 53-55.

Crider, A. B., Goethals, G. R., Kavanaugh, R. D., \& Solomon, P. R. (1986). Psychology (2nd ed.). Glenview, IL: Scott, Foresman.

Merrill, J. (1987). Levels of questioning and forms of feedback: Instructional factors in courseware design. Journal of Computer-Based Instruction, 14, 18-22.

MetzGER, R. L. (1985). Microcomputers in the small college psychology department. MicroPsych Network, 2, 6-8.

ORY, J. C. (1986). Ory testing program. Glenview, IL: Scott, Foresman.

PetTiJohn, T. F. (1985). Computer assisted programs for a general psychology course. MicroPsych Network, 2, 4-5.

ROPER, A. L. (1977). Feedback in computer-assisted instruction. Programmed Learning \& Educational Technology, 14, 43-49.

\section{APPENDIX A}

\section{Example of a Pair of Factual Questions}

A. A specific and involuntary response to a stimulus which does not require learning is called $a(n)$

1. conditioned response

2. operant response

3. reflex

4. conditioned association

B. Which of the following choices lists the conditioning phenomena in correct sequence?

1. Spontaneous recovery, acquisition, extinction

2. Acquisition, extinction, spontaneous recovery

3. Acquisition, spontaneous recovery, extinction

4. Extinction, acquisition, spontaneous recovery

Example of a Pair of Application-Type Questions

A. Billy, a child badly abused by his father, trembles and stammers in the presence of males. His behavior is an example of what process?

1. Generalization

2. Reinforcement

3. Spontaneous recovery

4. Submission

B. What process is involved when a young child smiles at familiar faces but cries when a strange face is present?

1. Generalization

2. Spontaneous recovery

3. Discrimination

4. Reinforcement

\section{APPENDIX B}

Examples of Questions and Feedback Formats

Part A

4. Every time Susie Q. starts to cry, her mother picks her up and comforts her. What schedule of reinforcement is the mother using?

1. Negative

2. Fixed

3. Continuous

4. Variable

\section{Part B}

Incorrect.

A variable schedule involves giving a reinforcement after an AVERAGE number of responses (variable ratio) or after an AVERAGE time interval (variable interval).

Your answer was 4. Try again-Enter a number (1-4):

Part C

You answered 3. Perfect!

Continuous schedule of reinforcement refers to the rewarding of a response EVERY time it is made, e.g., giving Susie comfort EVERY time she cried.

Do you want to know why one of the other answers is wrong?

Part D

Your answer was: 3 . The correct answer is: 3. Perfect!

Continous schedule of reinforcement refers to the rewarding of a response EVERY time it is made, e.g., giving Susie comfort EVERY time she cried. Negative reinforcement is not a SCHEDULE of reinforcement, but the removal of an aversive stimulus after some response has been made. A fixed schedule in operant conditioning is giving a reinforcer after a FIXED number of responses (ratio schedule) or after a FIXED interval of time (interval schedule). In this case Susie was reinforced EVERY time she cried. A variable schedule involves giving a reinforcement after an AVERAGE number of responses (variable ratio) or after an AVERAGE time interval (variable interval).

Hit a key to continue.

Note-Part A refers to the display of a sample stem and 4 choices. In both formats, the stem and choices were presented in the same display as the feedback. 
APPENDIX C

Questionnaire Distributed Before Second Practice Test

Please CIRCLE the most appropriate number to the question

\section{STRONGLY DISAGREE DISAGREE AGREE STRONGLY AGREE}

1. I read chapter 5 before the mini-test.

2. I read chapter $\mathbf{6}$ before the second mini-test.

3. I think the mini-tests help my study.

4. I think my score will improve on the next in-class exam.

5. I enjoy the mini-test procedure.

6. I felt discouraged after the first mini-test.

7. I have used or plan to use the information on the printout to review the chapters.

8. I studied harder for the second mini-test than for the first mini-test.

9. I would like to continue with mini-tests.

10. I would like mini-tests in other classes.

11. As a result of the first mini-test $I$ feel more motivated to study.

12. I think this mini-test procedure will help me learn what to identify when studying in the future.

13. I thought I knew the material on chapter 5 better than my score indicated.

14. How many questions do you think you will get right on tonight's mini-test? $\begin{array}{llll}1 & 2 & 3 & 4\end{array}$

$\begin{array}{llll}1 & 2 & 3 & 4\end{array}$

$\begin{array}{llll}1 & 2 & 3 & 4\end{array}$

$\begin{array}{llll}1 & 2 & 3 & 4\end{array}$

$\begin{array}{llll}1 & 2 & 3 & 4\end{array}$

$\begin{array}{llll}1 & 2 & 3 & 4\end{array}$

$\begin{array}{llll}1 & 2 & 3 & 4\end{array}$

$\begin{array}{llll}1 & 2 & 3 & 4\end{array}$

1234

$\begin{array}{llll}1 & 2 & 3 & 4\end{array}$

1234

$\begin{array}{llll}1 & 2 & 3 & 4\end{array}$

$\begin{array}{llll}1 & 2 & 3 & 4\end{array}$

2.8

7.5

3.2

3.7

out of 12

Mean Response

3.3

3.2

3.13 .1

$3.5 \quad 3.4$

$3.4 \quad 3.5$

$3.8 \quad 3.8$

$2.4 \quad 2.6$

$3.1 \quad 3.0$

$3.8 \quad 3.6$

$3.7 \quad 3.6$

$3.3 \quad 3.2$ 